\title{
Potential and Practical Implementations of Phase Sensitive Amplifiers for All-Optical Signal Regeneration
}

\author{
Francesca Parmigiani*, Joseph Kakande, Radan Slavik, Periklis Petropoulos, and David J. Richardson \\ Optoelectronics Research Centre, University of Southampton, Southampton, SO17 1BJ, UK \\ *frp@orc.soton.ac.uk
}

Phase noise introduced during transmission both from optical amplifiers and nonlinear interactions between channels represents a significant limiting factor to data transmission when advanced modulation formats such as (differential) phase-shift keying, (D)PSK, or quadrature phase shift keying, QPSK, signals are used to increase the network capacity [1]. Consequently, the development of all-optical techniques capable of eliminating phase (and ideally amplitude as well) noise from multi-level phase signals is of great interest. Phase regeneration of (D)PSK signals can be achieved directly by exploiting the phase-squeezing capability of phase sensitive amplifiers (PSAs) [2], which can be operated in the saturation regime to perform simultaneous amplitude regeneration. The main challenge in realising practical PSA-based regeneration is to stabilize and maintain a phase relationship between the PSA pump(s), the signal and any idlers present at the PSA input. This is complicated in practice by the fact that phase-encoded signals have a suppressed carrier. Moreover, even if the carrier can be extracted, it would generally incorporate any noise generated during data transmission. To overcome these issues a regenerative scheme has recently been demonstrated [3-5] which simultaneously allows carrier recovery and carrier noise suppression. The resulting cleaned carrier is then used to phase-lock the locally generated pumps with the incoming data prior to signal regeneration in a degenerate two-pump PSA [4-5]. In this scheme two important technologies were used: injection locking of semiconductor lasers to remove high frequency amplitude and phase noise during the carrier recovery process [6] and specialized highly nonlinear fibres (HNLFs) with an alumino-silicate core and a linear strain gradient applied along the length to increase the stimulated Brillouin scattering (SBS) threshold [7] in order to allow higher PSA gains. Figures 1 (a) and (b) show example constellation diagrams at the input/output of the regenerator for noisy input DPSK signals at 56Gbaud illustrating the regenerative performance achieved.

Whilst the regeneration of DPSK signals is a significant result in itself such schemes become of even greater interest if they can be scaled to more complex modulation formats, e.g. QPSK or higher. The regeneration of multi-level modulation format signals has very recently been proposed and demonstrated using more complex PSA configurations. Clearly, multi-level phase regeneration requires a staircase phase transfer function. In the concept proposed in [8-9], this is achieved by interfering the M-level optical PSK signal (where M is number of phase levels used) with its conjugated (M-1)th phase harmonic. For QPSK, the 3rd phase harmonic is used. Combining this newly generated harmonic with the signal leads to de-amplification of the phase noise states at $(\mathrm{N}-1) * 45$ degrees, while amplifying the signal states at $(\mathrm{N}-1) * 90$ degrees, for $\mathrm{N}=1: 4$ thereby providing phase regeneration. Figure 1 (c) and (d) shows an example of constellation diagrams at the input/output of the regenerator for QPSK signals at 56Gbaud (again noisy input signals were used). This basic concept can be readily extended to higher level phase encoded formats.
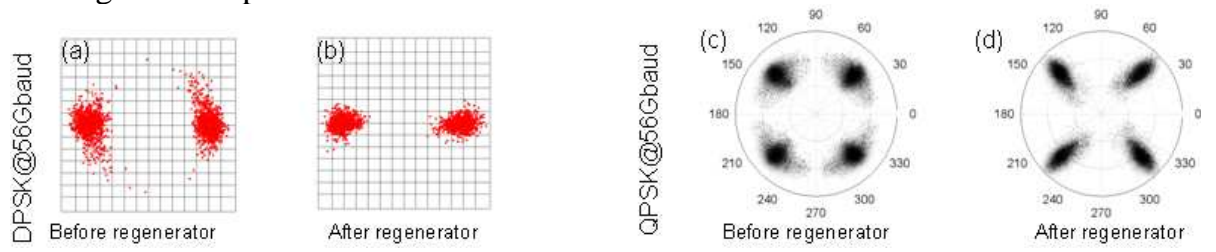

Fig. 1 Examples of constellation diagrams at 56Gbaud at the regenerator input (a,c) and output (b, d) for DPSK and QPSK signals, respectively. The measurements were taken with the EXFO PSO200 Lightwave Signal Analyzer.

\section{References}

[1] J. P. Gordon and L. F. Mollenauer, "Phase noise in photonic communications systems using linear amplifiers," Opt. Lett. 15(23), 13511353 (1990).

[2] K. Croussore, et al., "Demonstration of phase-regeneration of DPSK signals based on phase-sensitive amplification," Opt. Express, 13, 3945-3950 (2005).

[3] Weerasuriya, et al. , "Generation of frequency symmetric signals from a BPSK input for phase sensitive amplification," OFC, pp.1-3, 2125 March 2010.

[4] R. Slavík et al, “All-optical phase and amplitude regenerator for next-generation telecommunications systems,” Nature Photonics. 4, 690695 (2010).

[5] R. Slavík et al, "Robust design of all-optical PSK regenerator based on phase sensitive amplification," OFC 2011.

[6] S. Sygletos et al., "Phase locking and carrier extraction scheme for phase sensitive amplification," ICTON, paper Mo.C1.3, June 2010. [7] L. Grüner-Nielsen et al., "A Silica Based Highly Nonlinear Fibre with Improved Threshold for Stimulated Brillouin Scattering," paper Tu.4.D.3, ECOC 2010.

[8] J. Kakande et al, "First demonstration of all-optical QPSK signal regeneration in a novel multi-format phase sensitive amplifier," ECOC 2010 Turin 19-23, Italy, PD 3.3, Sep 2010.

[9] J. Kakande et al, "First QPSK Phase and Amplitude Regeneration at 56 Gbaud in a Novel Idler-Free Non-Degenerate Phase Sensitive Amplifier," OFC 2011. 